\title{
Engineering intracellular malonyl-CoA availability in microbial hosts and its impact on polyketide and fatty acid synthesis
}

\author{
Lars Milke $^{1}$ (D) Jan Marienhagen ${ }^{1,2,3}$ (D) \\ Received: 19 February 2020 / Revised: 9 April 2020 / Accepted: 21 April 2020 / Published online: 8 May 2020 \\ (C) The Author(s) 2020
}

\begin{abstract} productivity and product range, with a focus on polyketides and other malonyl-CoA-dependent products.

Key Points

- Malonyl-CoA is the central building block of polyketide synthesis.

- Increasing acetyl-CoA supply is pivotal to improve malonyl-CoA availability.

- Improved acetyl-CoA carboxylase activity increases availability of malonyl-CoA.

- Fatty acid synthesis as an ambivalent target to improve malonyl-CoA supply.
\end{abstract}

Malonyl-CoA is an important central metabolite serving as the basic building block for the microbial synthesis of many pharmaceutically interesting polyketides, but also fatty acid-derived compounds including biofuels. Especially Saccharomyces cerevisiae, Escherichia coli, and Corynebacterium glutamicum have been engineered towards microbial synthesis of such compounds in recent years. However, developed strains and processes often suffer from insufficient productivity. Usually, tightly regulated intracellular malonyl-CoA availability is regarded as the decisive bottleneck limiting overall product formation. Therefore, metabolic engineering towards improved malonyl-CoA availability is essential to design efficient microbial cell factories for the production of polyketides and fatty acid derivatives. This review article summarizes metabolic engineering strategies to improve intracellular malonyl-CoA formation in industrially relevant microorganisms and its impact on

Keywords Malonyl-CoA $\cdot$ Metabolic engineering $\cdot$ Polyketide $\cdot$ Fatty acid $\cdot$ Biofuel

\section{Introduction}

Polyketides are an outstanding group of secondary metabolites with regard to their structural diversity and the number of their clinical applications (Hopwood 2009; Osbourn and Lanzotti 2009; Wink 2010; Robertsen and Musiol-Kroll 2019). Not only does this group comprise antibiotics (e.g., erythromycin A, azithromycin), anticancer drugs (e.g., enediynes), and drugs for the treatment of cardiovascular diseases (e.g., lovastatin) but also important immunosuppressants such as rapamycin. Of the 7,000

Jan Marienhagen

j.marienhagen@fz-juelich.de

1 Institute of Bio- and Geosciences, IBG-1: Biotechnology, Forschungszentrum Jülich GmbH, 52425 Jülich, Germany

2 Institute of Biotechnology, RWTH Aachen University, Worringer Weg 3, 52074 Aachen, Germany

3 Bioeconomy Science Center (BioSC), Forschungszentrum Jülich GmbH, 52425 Jülich, Germany polyketides known, more than 20 have been commercialized, which resembles a "success rate" of $0.3 \%$ (Weissman and Leadlay 2005). In total, polyketide-derived pharmaceuticals make up $20 \%$ of the top-selling drugs, generating a worldwide revenue of over $€ 14$ billion annually (Weissman and Leadlay 2005). Additionally, polyketides include polyphenols comprising flavonoids and stilbenes such as naringenin and resveratrol (Fig. 1). These molecules provide a plethora of beneficial effects on human health including antioxidant, anti-inflammatory, or anti-cancerous characteristics (Pandey and Rizvi 2009). Furthermore, a positive effect in prevention or treatment of cardiovascular and neurodegenerative diseases, but also obesity and diabetes, was described for selected compounds (Khurana et al. 2013). In this context, resveratrol is probably the most prominent example, which is successfully marketed as dietary supplement (Catalgol et al. 2012). In total, the global nutraceutical market was accounted for $\$ 379$ billion in 2017 (Stratistics Market Research Consulting Pvt Ltd 2018).

Polyketides are ubiquitous metabolites found in bacteria, fungi, and plants. Despite their structural diversity, all polyketides 


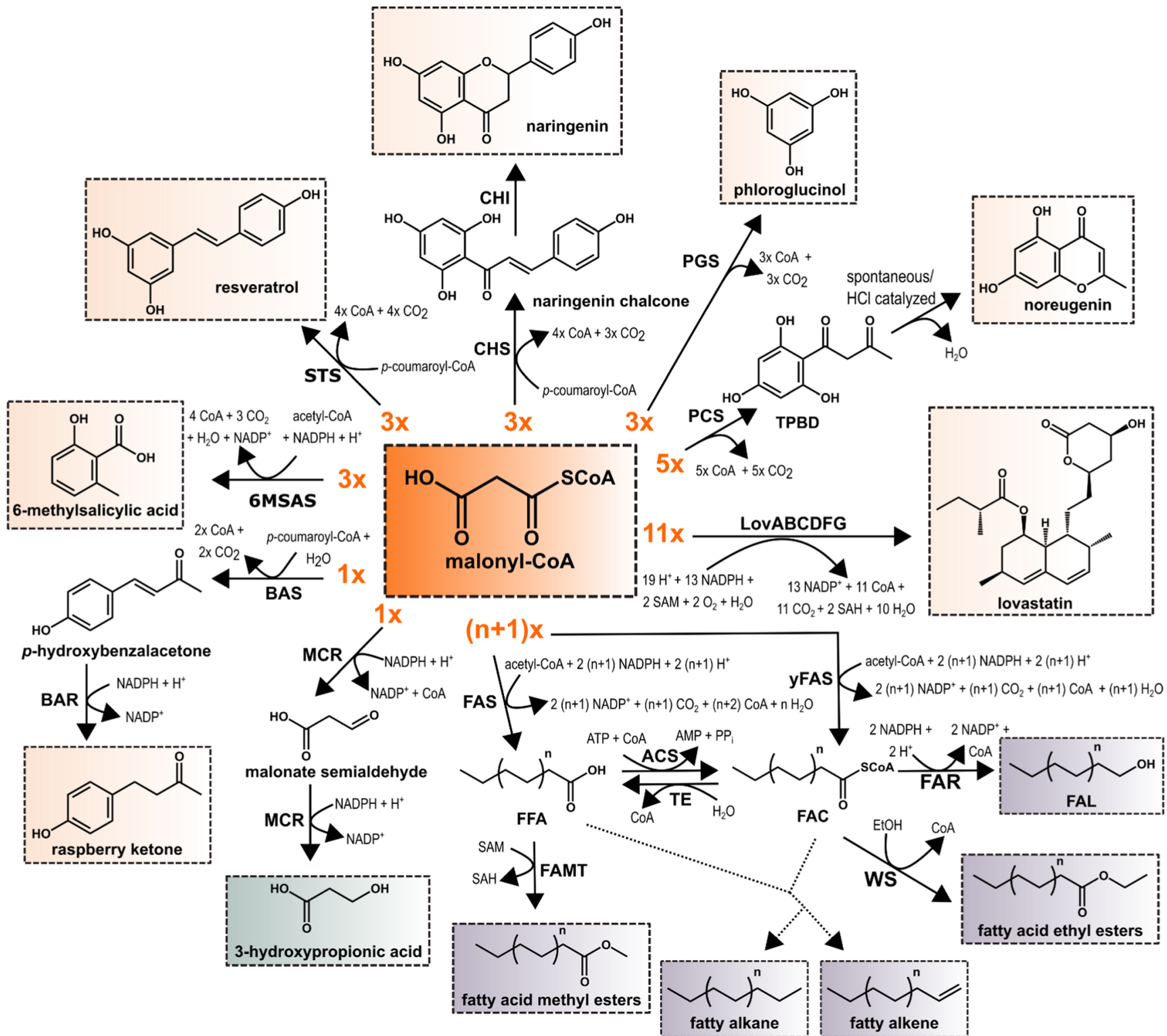

Fig. 1 Overview of different microbially synthesized malonyl-CoAdependent molecules. 6MSAS 6-methylsalicylic acid synthase, ACS acyl-CoA synthetase, BAR benzalacetone reductase, BAS benzalacetone synthase, CHI chalcone isomerase, CHS chalcone synthase, FAC fattyacyl-CoA, FAL fatty acid alcohol, FAMT fatty acid $O$-methyltransferase, FAR fatty-acyl-CoA reductase, FAS fatty acid synthase, FFA free fatty acid, LovABCDFG lovastatin megasynthase complex from Aspergillus terreus, MCR malonyl-CoA reductase, PCS pentaketide chromone synthase, PGS phloroglucinol synthase, SAH $S$-adenosyl-homocysteine,

are synthesized by iterative, decarboxylative Claisen condensation of acyl-CoA units catalyzed by polyketide synthases (PKSs). Based on their overall architectural structure, which defines starter unit selection, chain elongation, degree of reduction, and cyclization patterns, PKS are classified into three types (types I-III) (Austin and Noel 2003). Whereas the type I family comprises large, multifunctional polypeptides with multiple, catalytically active domains, type II family PKSs typically are aggregates of
SAM $S$-adenosylmethionine, STS stilbene synthase, TE fatty-acyl-CoA thioesterase, TPBD 1-(2,4,6-tri-hydroxyphenyl)butane-1,3-dione, WS wax ester synthase, yFAS yeast fatty acid synthase/fatty-acyl-CoA synthase. Light orange boxes indicate polyketide products, light green boxes carboxylic acids, and light purple boxes fatty acid-derived biofuels. For fatty acid synthesis, $n$ represents the number of malonyl-CoA-derived $\mathrm{C} 2$ units incorporated into the growing acyl-chain between the acetyl-CoAderived starter unit (terminal methyl group) and the final malonyl-CoAderived extender unit (terminal carboxyl group), which accounts for +1

dissociable, monofunctional enzymes (Hertweck 2009). Depending on the PKS type, the individual domains or the monofunctional enzymes provide acyltransferase, acyl carrier protein, $\beta$-ketosynthase $(\mathrm{KS})$, and thioesterase functions. $\beta$ keto processing capabilities including dehydratase, enoylreductase, and ketoreductase activities are optional. In accordance with the fatty acid synthase (FAS) classification, type I PKSs predominantly occur in fungi and animals, whereas type II 
PKSs are commonly found in prokaryotes, in particular actinomycetes. In contrast, type III PKSs - also referred to as chalcone/ stilbene synthases (CHS/STS) - are KS-like homodimers usually to be found in plants, but also bacteria and some fungi. Despite their structural simplicity, type III PKSs can perform starter unit selection, catalyze (iterative) chain elongation, and control cyclization patterns. However, independent from this classification, most PKSs consume malonyl-CoA molecules as extender units, which are successively added to growing $\beta$-ketoacyl chains (Chan et al. 2009). Departing from this, the type I PKS from Saccharopolyspora erythraea synthesizing the 6deoxyerythronolide B scaffold of erythromycin A condenses one propionyl-CoA starter unit with six methylmalonyl-CoA extender units (Rawlings 2001).

Depending on the respective polyketide product, very different malonyl-CoA quantities are required. Whereas the synthesis of the flavoring phenylbutanoid raspberry ketone requires only one malonyl-CoA molecule, three malonyl-CoA units are typically needed for the synthesis of plant polyphenols such as resveratrol and naringenin, and during lovastatin biosynthesis eleven malonyl-CoA molecules are consumed (Fig. 1) (Campbell and Vederas 2010; Shimokawa et al. 2012; Milke et al. 2018). In most organisms, malonyl-CoA is exclusively synthesized by acetyl-CoA carboxylation catalyzed by acetyl-CoA carboxylases (ACC). This enzyme complex comprises three different domains catalyzing two distinct reaction steps (Cronan and Waldrop 2002). Initially, an ATPdependent biotin carboxylase (BC) domain catalyzes the carboxylation of biotin with bicarbonate, which is covalently attached to a biotin carboxyl carrier protein (BCCP) domain over a lysine residue, forming carboxybiotin. A flexible biotin arm shuttles the carboxybiotin from the $\mathrm{BC}$ domain to the carboxyltransferase (CT) domain required for the second half reaction in which the carboxygroup is transferred to acetylCoA forming malonyl-CoA. However, the role of malonylCoA as building block for secondary metabolite synthesis plays only a minor role in the cellular metabolism. Primarily, malonyl-CoA serves as extender unit for the synthesis of fatty acids, constituting the hydrophobic domain of membrane lipids (Cronan and Thomas 2009). Noteworthy, fatty acidderived alcohols (FAL), alkyl esters (FAME, FAEE), and alkanes/alkenes are considered to be promising secondgeneration biofuels as they provide similar chemical properties as petroleum-based fuels, allowing to readily replace them (Fig. 1) (Sheng and Feng 2015; Hu et al. 2019). In total, the global market for natural fatty acids had an value of nearly \$13.5 billion in 2018 (BCC Research LLC 2019).

Current efforts towards the transformation of a fossil-based economy to a more sustainable bio-based economy have drawn attention to the microbial synthesis of both polyketides and fatty acids (Takeno et al. 2013; Yang et al. 2018; Hu et al. 2019). Unfortunately, the strictly regulated intracellular malonyl-CoA availability in well-established microbial platform organisms was identified as decisive bottleneck limiting overall product formation (Marienhagen and Bott 2013; Janßen and Steinbüchel 2014; Palmer and Alper 2018). Therefore, numerous studies focused on metabolic engineering of microorganisms towards improved malonyl-CoA availability. In this review article, we provide an overview of advancements in engineering relevant microbial hosts such as Escherichia coli, Saccharomyces cerevisiae, and Corynebacterium glutamicum towards improved malonylCoA availability and its effect on product range and productivity.

\section{Increasing acetyl-CoA supply is essential for improving intracellular malonyl-CoA availability}

Typically, tailoring the central carbon metabolism towards increased availability of acetyl-CoA as direct malonyl-CoA precursor molecule is beneficial for malonyl-CoA synthesis and all malonyl-CoA-derived products. In this context, several metabolic engineering strategies aiming for both improved synthesis and reduced consumption of acetyl-CoA have been developed (Fig. 2).

Recently, it could be shown that focusing the glycolytic flux towards acetyl-CoA by partial elimination of anaplerotic pyruvate carboxylation contributes to the malonyl-CoAdependent synthesis of noreugenin $(53 \mathrm{mg} / \mathrm{L})$, a pentaketide from the medical plant Aloe arborescens, in C. glutamicum (Milke et al. 2019b). In E. coli, increasing flux through the glycolytic pathway by overexpressing genes encoding the phosphoglycerate kinase and pyruvate dehydrogenase enabled the accumulation of naringenin $(474 \mathrm{mg} / \mathrm{L})(\mathrm{Xu}$ et al. 2011). Also in E. coli, increased glucose utilization due to the implementation of an alternative, PTS-system-independent glucose facilitator protein from Zymomonas mobilis contributed to an increased acetyl-CoA-dependent synthesis of $N$ acetylglutamate (Zhang et al. 2019). Likewise, deregulated expression of iolT1 in C. glutamicum, encoding a glucose/ myo-inositol permease, improved glucose uptake, which in turn allowed for increased polyketide and hydroxybenzoic acid synthesis (Brüsseler et al. 2018; Kallscheuer and Marienhagen 2018; Milke et al. 2019b).

In E. coli, deletion of acetyl-CoA consuming acetate and ethanol forming pathways resulted in a 15 -fold improved malonyl-CoA availability, allowing for the synthesis of phloroglucinol (1280 mg/L) (Fig. 1) (Zha et al. 2009). Besides preventing acetate formation under aerobic conditions from acetyl-CoA, expression of a gene encoding an acetylCoA synthetase enabled recycling of acetate to increase the acetyl-CoA pool (Wu et al. 2017). This strategy was applied in E. coli to increase acetyl-CoA availability 3.7 -fold, ultimately increasing medium chain fatty acid synthesis to $683 \mathrm{mg} / \mathrm{L}$. In 


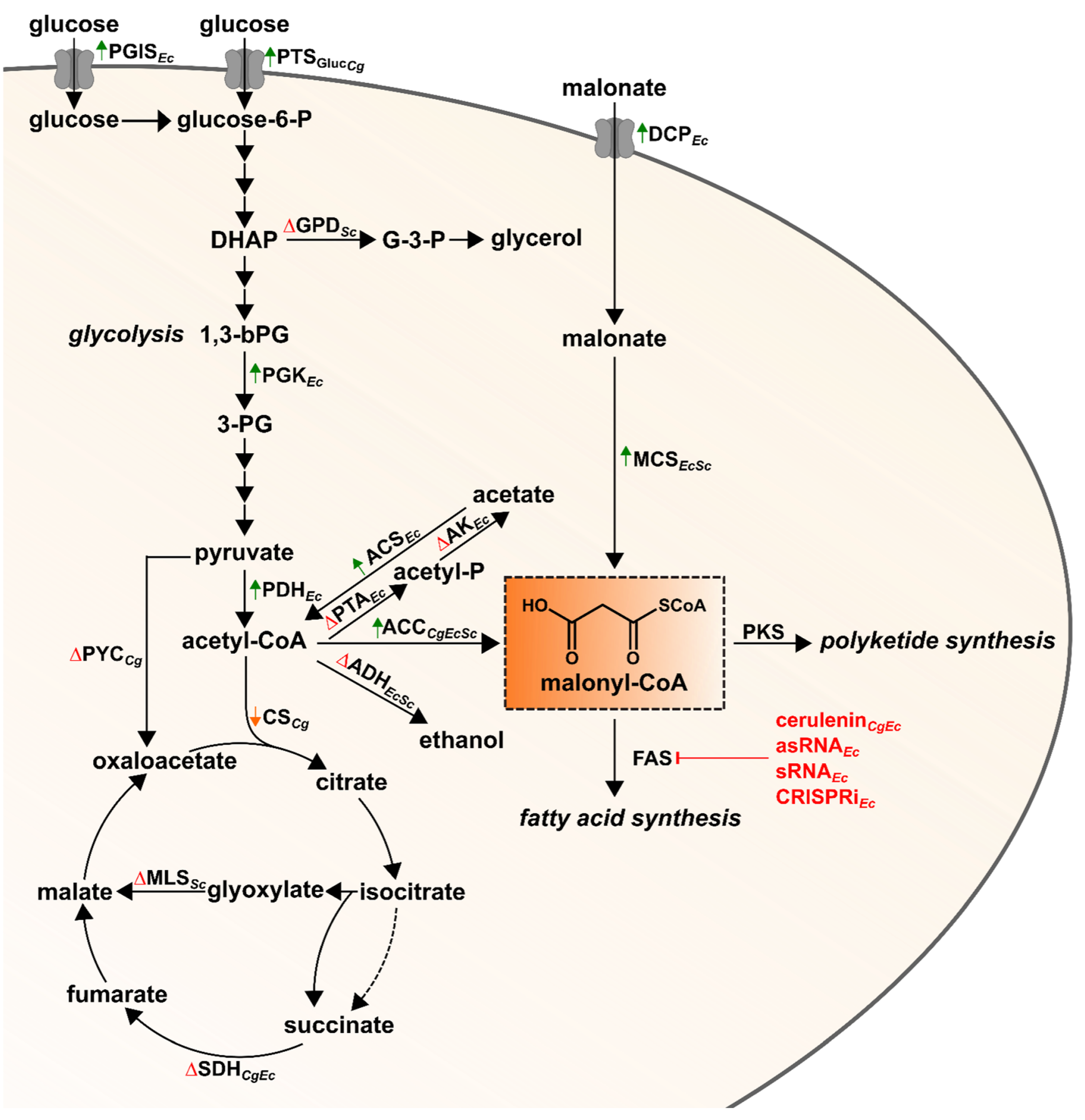

Fig. 2 Overview of metabolic engineering strategies towards improved intracellular malonyl-CoA availability in microorganisms. 1,3-bPG 1,3bisphosphoglycerate, 3-PG 3-phosphoglycerate, ACC acetyl-CoA carboxylase ACS acetyl-CoA synthetase, ADH alcohol dehydrogenase, AK acetate kinase, asRNA antisense RNA, CRISPRi clustered regularly interspaced short palindromic repeats interference, CS citrate synthase, DHAP dihydroxyacetone phosphate, DCP dicarboxylate carrier protein, FAS fatty acid synthase, G-3-P glycerol-3-phosphate, GPD glycerol-3phosphate dehydrogenase, MCS malonyl-CoA synthetase, MLS malate synthase, PDH pyruvate dehydrogenase complex, PGIS PTSindependent glucose import system, PGK phosphoglycerate kinase,

S. cerevisiae, deletion of genes encoding alcohol dehydrogenases and glycerol-3-phosphate dehydrogenases, partially or
PKS polyketide synthase, PTA phosphate acetyltransferase, PTS $_{\mathrm{Gluc}}$ glucose-specific phosphotransferase system, PYC pyruvate carboxylase, SDH succinate dehydrogenase complex, sRNA small regulatory RNA. Increased or heterologous enzyme activity is indicated by an upward green arrow $(\uparrow)$, reduced enzyme activity by a downward orange arrow $(\downarrow)$, and eliminated enzyme reactions by a red delta $(\Delta)$. Metabolic engineering strategies presented in this review are assigned to the particular microorganism by subscripted abbreviations for C. glutamicum $\left(_{C g}\right)$, E. $\operatorname{coli}\left({ }_{E c}\right)$, and $S$. cerevisiae $\left({ }_{S c}\right)$

completely inhibiting ethanol and glycerol biosynthesis, ultimately improved acetyl-CoA availability two-fold, 
contributing to the synthesis of over $100 \mathrm{mg} / \mathrm{L} n$-butanol in high cell-density fermentations (Lian et al. 2014). Furthermore, inactivation of the alcohol dehydrogenase ADH1 in yeast was demonstrated to increase fatty acid synthesis 1.9-fold reaching titers of up to $120 \mathrm{mg} / \mathrm{L}$ (Li et al. 2014).

However, oxidation in the tricarboxylic acid (TCA) cycle or glyoxylate shunt is the main fate of acetyl-CoA. In this context, disruption of the glyoxylate shunt involved in the transport of acetyl-CoA between the cytosol and cellular compartments in S. cerevisiae was demonstrated to increase cytosolic acetyl-CoA availability for the biosynthesis of $n$-butanol (Lian et al. 2014). Furthermore, sophisticated metabolic models for $E$. coli and $C$. glutamicum were used to predict multiple gene knock-out combinations, which would increase acetyl-CoA availability. In this context, interruption of the TCA cycle by deleting succinate dehydrogenase complex encoding genes was demonstrated to reduce acetyl-CoA consumption, allowing for the two-fold increased naringenin synthesis in both microorganisms (Fowler et al. 2009; Hartmann et al. 2017; Milke et al. 2019a). However, interrupting the TCA cycle has a negative impact on growth rate and biomass formation on industrially relevant carbon- and energy sources, especially in C. glutamicum, which puts this particular modification into question with regard to its overall beneficial impact. Alternatively, to reduce acetyl-CoA consumption via the TCA cycle in C. glutamicum, activity of the pace-making citrate synthase (CS) was reduced stepwise to $5.5 \%$ compared with the wild-type CS activity by exchanging the native promotor of the CS-encoding gltA gene (Milke et al. 2019a, b). Interestingly, overall biomass formation was barely distinguishable from the parental C. glutamicum strain with wildtype CS activity, although the growth rate was significantly reduced. Eventually, naringenin synthesis was increased tenfold to $19 \mathrm{mg} / \mathrm{L}$, indicating an increased intracellular availability of malonyl-CoA, which was later verified by LC-MS/MS quantification.

\section{High ACC activity improves malonyl-CoA availability}

As malonyl-CoA is solely derived from the carboxylation of acetyl-CoA in E. coli, S. cerevisiae, and C. glutamicum, increasing ACC activity is important to make use of the available acetyl-CoA pool. Whereas bacterial and plant chloroplastic ACCs are organized as complexes of distinct dissociable polypeptides, mammalian, fungal, and plant cytosolic ACCs are single polypeptide chains possessing all catalytically active domains (Cronan and Waldrop 2002; Tong 2005). A widely used strategy to increase ACC activity is expression of heterologous genes encoding ACC subunits. Heterologous expression of genes encoding the heterotetrameric ACC and the biotin ligase from Photorhabdus luminescens in E. coli improved the microbial synthesis of the plant polyphenol pinocembrin seven-fold, allowing for a maximal product titer of $196 \mathrm{mg} / \mathrm{L}$ (Leonard et al. 2007). Interestingly, the ACC of C. glutamicum requires only two subunits (AccB1, AccD1) instead of four subunits for catalytic activity, rendering this enzyme an often exploited alternative to heterotetrameric ACCs for improving intracellular malonyl-CoA availability (Miyahisa et al. 2005; Gande et al. 2007; Cheng et al. 2016). However, expression of ACC encoding genes was not always successful in terms of microbial polyphenol synthesis (van Summeren-Wesenhagen and Marienhagen 2015). Interestingly, although originating from this particular organism, episomal overexpression of $a c c B C$ and $a c c D 1$ hardly increased malonyl-CoA-dependent naringenin biosynthesis in C. glutamicum itself (Milke et al. 2019a). Thus, metabolic engineering of $C$. glutamicum to increase transcription of the genome-encoded $a c c B C$ and $a c c D 1$ genes could be a more promising strategy for improving malonyl-CoA supply.

Similar to most microorganisms, fatty acid synthesis in C. glutamicum is tightly regulated on the transcriptional level (Schujman et al. 2006; Nickel et al. 2010). In the case of C. glutamicum, transcription of the ACC genes accBC and $a c c D 1$, but also of the FAS encoding genes fas $A$ and $\operatorname{fas} B$, is inhibited by the TetR-type transcriptional repressor FasR, which binds to a highly conserved fas $O$ motif upstream of the regulated open reading frames. Acyl-CoA thioesters (oleoyl-CoA and palmitoyl-CoA) are regarded as effectors interacting with FasR (Irzik et al. 2014). In this context, it was shown that a FasR-S20N mutant, characterized by increased transcription levels of $a c c D 1$, fas $A$, and fas $B$, dramatically improved the production of malonyl-CoA-dependent fatty acids, mainly oleic acid (Takeno et al. 2013). This particular amino acid substitution was postulated to either interfere with FasR-acyl-CoA complex formation or binding of the repressor-effector complex to the fas $O$ motifs. Alternatively, in-frame deletion of the FasR-encoding gene fas $R$ also increased oleic acid production (Takeno et al. 2013). However, this particular deletion was not beneficial for naringenin synthesis using $C$. glutamicum, probably due to the increased malonyl-CoA consumption by FASs (FasA and FasB), encoded by the FasR-controlled genes fas $A$ and fas $B$ (Milke et al. 2019a). In a more sophisticated approach, mutation of individual nucleotides within the fas $O$ motifs of the $a c c B C$ and $a c c D 1$ promoters repealed FasR-mediated regulation, allowing for an almost tripled intracellular malonyl-CoA concentration in C. glutamicum (Milke et al. 2019b). The constructed $C$. glutamicum strain did not only enable synthesis of the plant pentaketide noreugenin but also allowed for the formation malonyl-CoA-dependent polyketides 6methylsalicylic acid (6-MSA) as well as different biotechnologically interesting flavoring phenylbutanoids (Kallscheuer et al. 2019; Milke et al. 2020). In principle, transcriptional deregulation of genomic ACC encoding genes appears to be 
feasible strategy for all microbial hosts, provided that such a regulation is present. Additionally, omission of auxiliary plasmids for improving ACC activity could not only lower the metabolic burden but also reduce overall process costs ( $\mathrm{Wu}$ et al. 2016).

In S. cerevisiae, ACC activity is not only transcriptionally but also post-translationally regulated. At the transcriptional level, replacement the native promotor of the $A C C 1$ gene with the strong constitutive TEF1 promotor improved 6-MSA production yielding up to $554 \mathrm{mg} / \mathrm{L}$ in bioreactor cultivations (Wattanachaisaereekul et al. 2008). In terms of posttranslational regulation, removal of the Snfl-dependent phosphorylation of Acc1 improved malonyl-CoA supply, allowing for an increased synthesis of fatty acid ethyl esters and 3hydroxypropionic acid (Shi et al. 2014).

Alternatively, the ACC-independent formation of malonylCoA using the malonate assimilation pathway from Rhizobium trifolii increased microbial synthesis of the polyphenolic polyketide pinocembrin up to 15 -fold (final titer $480 \mathrm{mg} / \mathrm{L}$ ) using E. coli (An and Kim 1998; Leonard et al. 2008; Wu et al. 2013). In S. cerevisiae, overexpression of $A A E 13$, encoding the malonyl-CoA synthetase from Arabidopsis thaliana, significantly improved microbial resveratrol and fatty acid synthesis (Wang et al. 2014). Noteworthy, a temperature-sensitive $A C C 1$ variant could be complemented by this heterologous pathway.

\section{Increasing malonyl-CoA availability for polyketide production by inhibition of fatty acid synthesis}

In general, the strategies described above are applicable for microbial synthesis of polyketides and fatty acids. However, in terms of microbial polyketide synthesis, fatty acids are regarded as undesired byproducts withdrawing malonyl$\mathrm{CoA}$ and thus limiting overall polyketide formation (Milke et al. 2018). Therefore, endogenous fatty acid synthesis represents a promising target to increase malonyl-CoA availability for polyketide synthesis.

In order to reduce undesired malonyl-CoA consumption, supplementation of the potent FAS inhibitor cerulenin was widely used, which allowed for an increased synthesis of polyphenolic polyketides in E. coli and C. glutamicum (Leonard et al. 2008; Lim et al. 2011; Santos et al. 2011; van Summeren-Wesenhagen and Marienhagen 2015; Kallscheuer et al. 2016). However, drawback of cerulenin supplementation is the non-selective and irreversible inhibition of FASs by covalently binding to a conserved active site cysteine residue of the KS domain, which almost instantaneously stops microbial growth due to rapid fatty acid depletion (Price et al. 2001). Furthermore, not only the KS domain of FASs but also the KS domain of PKSs could be inhibited, possibly limiting, or even preventing the desired polyketide formation (Ferrer et al. 1999). Additionally, cerulenin is very expensive, rendering application of this antibiotic unsuitable for any large-scale applications (Milke et al. 2019a).

Alternatively, post-transcriptional downregulation of genes involved in fatty acid synthesis in $E$. coli using antisense RNAs, small regulatory RNAs, or CRISPR interference was used to improve malonyl-CoA-dependent synthesis of the polyphenolic polyketides naringenin, resveratrol, and pinosylvin (Wu et al. 2014; Cress et al. 2015; Wu et al. 2015; Yang et al. 2015; Liang et al. 2016; Yang et al. 2018).

\section{Untargeted strain evolution towards improved malonyl-CoA availability}

In addition to the rational metabolic engineering strategies described, undirected approaches involving the generation of random genetic diversity and subsequent screening were also successfully used to isolate microbial strain variants with increased intracellular malonyl-CoA availability. In this context, transcriptional biosensors, linking production phenotypes to fluorescence output signals in combination with fluorescence activated cell sorting (FACS), represent powerful highthroughput tools for rapidly isolating mutants from genetically diverse strain libraries (Flachbart et al. 2019). Available malonyl-CoA-responsive biosensors are based on the transcriptional repressor FapR, which is involved in regulation of fatty acid synthesis in Bacillus subtilis (Schujman et al. 2006; Xu et al. 2014b; Johnson et al. 2017). In addition to the construction of synthetic regulatory circuits dynamically controlling gene expression, a FapR-based transcriptional biosensor was used to identify novel gene targets in S. cerevisiae for improved malonyl-CoA availability (Xu et al. 2014a; Li et al. 2015; Johnson et al. 2017). In a FACS screening campaign, isolated $S$. cerevisiae variants showing increased expression of the genes PMP1 and TPII involved in biotin uptake and ATP availability accumulated significantly more 3hydroxypropionic acid ( $\mathrm{Li}$ et al. 2015). Alternatively, colorimetric screening assays indicating improved malonyl-CoA availability can be used. For this purpose, the malonyl-CoAdependent synthesis of the red-colored polyketide flaviolin, which is catalyzed by the type III PKS RppA, found an application (Yang et al. 2018). In the microtiter plate format, this assay was successfully used to screen a synthetic small regulatory RNA library, identifying 14 knockdown targets in E. coli. Furthermore, the RppA biosensor was also demonstrated to be functional in Pseudomonas putida and C. glutamicum. In combination with miniaturized and automated adaptive laboratory evolution experiments, this screening approach represents a promising strategy for the untargeted optimization of rationally engineered strains. 
Code availability Not applicable.

Authors' contributions LM and JM wrote the manuscript. Both authors read and approved the manuscript.

Funding information Open Access funding provided by Projekt DEAL. This work was funded by the Bioeconomy Science Center (BioSC) as part of the FocusLab project "HyImPAct - Hybrid processes for important precursor and active pharmaceutical ingredients" (Grant No.: 325 - 400 002 13) and by the German Federal Ministry of Education and Research (BMBF, Grant No.: 031B0918A) as part of the project "BioökonomieREVIER."

Data availability Not applicable.

\section{Compliance with ethical standards}

Conflict of interest The authors declare that they have no conflict of interest.

Ethical approval Not applicable.

Consent to participate Not applicable.

Consent for publication Not applicable.

Open Access This article is licensed under a Creative Commons Attribution 4.0 International License, which permits use, sharing, adaptation, distribution and reproduction in any medium or format, as long as you give appropriate credit to the original author(s) and the source, provide a link to the Creative Commons licence, and indicate if changes were made. The images or other third party material in this article are included in the article's Creative Commons licence, unless indicated otherwise in a credit line to the material. If material is not included in the article's Creative Commons licence and your intended use is not permitted by statutory regulation or exceeds the permitted use, you will need to obtain permission directly from the copyright holder. To view a copy of this licence, visit http://creativecommons.org/licenses/by/4.0/.

\section{References}

An JH, Kim YS (1998) A gene cluster encoding malonyl-CoA decarboxylase (MatA), malonyl-CoA synthetase (MatB) and a putative dicarboxylate carrier protein (MatC) in Rhizobium trifolii - cloning, sequencing, and expression of the enzymes in Escherichia coli. Eur J Biochem 257:395-402. https://doi.org/10.1046/j.1432-1327.1998. 2570395.x

Austin MB, Noel JP (2003) The chalcone synthase superfamily of type III polyketide synthases. Nat Prod Rep 20:79-110. https://doi.org/10. 1039/b100917f

BCC Research LLC (2019) Oleochemical fatty acids: global markets to 2023. In: Oleochemical Fat. Acids Glob. Mark. to 2023. https:// www.bccresearch.com/market-research/chemicals/oleochemicalfatty-acids-global-markets.html. Accessed 9 Jan 2020

Brüsseler C, Radek A, Tenhaef N, Krumbach K, Noack S, Marienhagen J (2018) The myo-inositol/proton symporter IolT1 contributes to Dxylose uptake in Corynebacterium glutamicum. Bioresour Technol 249:953-961. https://doi.org/10.1016/j.biortech.2017.10.098

Campbell CD, Vederas JC (2010) Biosynthesis of lovastatin and related metabolites formed by fungal iterative PKS enzymes. Biopolymers 93:755-763. https://doi.org/10.1002/bip.21428
Catalgol B, Batirel S, Taga Y, Ozer NK (2012) Resveratrol: French paradox revisited. Front Pharmacol 3:1-18. https://doi.org/10.3389/ fphar.2012.00141

Chan YA, Podevels AM, Kevany BM, Thomas MG (2009) Biosynthesis of polyketide synthase extender units. Nat Prod Rep 26:90-114. https://doi.org/10.1039/b801658p

Cheng Z, Jiang J, Wu H, Li Z, Ye Q (2016) Enhanced production of 3hydroxypropionic acid from glucose via malonyl-CoA pathway by engineered Escherichia coli. Bioresour Technol 200:897-904. https://doi.org/10.1016/j.biortech.2015.10.107

Cress BF, Toparlak OD, Guleria S, Lebovich M, Stieglitz JT, Englaender JA, Jones JA, Linhardt RJ, Koffas MAG (2015) CRISPathBrick: modular combinatorial assembly of type II-A CRISPR arrays for dCas9-mediated multiplex transcriptional repression in E. coli. ACS Synth Biol 4:987-1000. https://doi.org/10.1021/acssynbio. 5 b00012

Cronan JE, Thomas J (2009) Bacterial fatty acid synthesis and its relationships with polyketide synthetic pathways. Methods Enzymol 459:395-433. https://doi.org/10.1016/S0076-6879(09)04617-5

Cronan JE, Waldrop GL (2002) Multi-subunit acetyl-CoA carboxylases. Prog Lipid Res 41:407-435. https://doi.org/10.1016/s01637827(02)00007-3

Ferrer J-L, Jez JM, Bowman ME, Dixon RA, Noel JP (1999) Structure of chalcone synthase and the molecular basis of plant polyketide biosynthesis. Nat Struct Biol 6:775-784. https://doi.org/10.1038/11553

Flachbart LK, Sokolowsky S, Marienhagen J (2019) Displaced by deceivers: prevention of biosensor cross-talk is pivotal for successful biosensor-based high-throughput screening campaigns. ACS Synth Biol 8:1847-1857. https://doi.org/10.1021/acssynbio.9b00149

Fowler ZL, Gikandi WW, Koffas MAG (2009) Increased malonyl coenzyme A biosynthesis by tuning the Escherichia coli metabolic network and its application to flavanone production. Appl Environ Microbiol 75:5831-5839. https://doi.org/10.1128/AEM.00270-09

Gande R, Dover LG, Krumbach K, Besra GS, Sahm H, Oikawa T, Eggeling L (2007) The two carboxylases of Corynebacterium glutamicum essential for fatty acid and mycolic acid synthesis. J Bacteriol 189:5257-5264. https://doi.org/10.1128/JB.00254-07

Hartmann A, Vila-Santa A, Kallscheuer N, Vogt M, Julien-Laferrière A, Sagot M-F, Marienhagen J, Vinga S (2017) OptPipe - a pipeline for optimizing metabolic engineering targets. BMC Syst Biol 11:1-9. https://doi.org/10.1186/s12918-017-0515-0

Hertweck C (2009) The biosynthetic logic of polyketide diversity. Angew Chem Int Ed 48:4688-4716. https://doi.org/10.1002/anie. 200806121

Hopwood DA (ed) (2009) Complex enzymes in microbial natural product biosynthesis, part B: polyketides, aminocoumarins and carbohydrates, first. Academic Press, Cambridge

$\mathrm{Hu}$ Y, Zhu Z, Nielsen J, Siewers V (2019) Engineering Saccharomyces cerevisiae cells for production of fatty acid-derived biofuels and chemicals. Open Biol 9:190049. https://doi.org/10.1098/rsob. 190049

Irzik K, van Ooyen J, Gätgens J, Krumbach K, Bott M, Eggeling L (2014) Acyl-CoA sensing by FasR to adjust fatty acid synthesis in Corynebacterium glutamicum. J Biotechnol 192:96-101. https:// doi.org/10.1016/j.jbiotec.2014.10.031

Janßen HJ, Steinbüchel A (2014) Fatty acid synthesis in Escherichia coli and its applications towards the production of fatty acid based biofuels. Biotechnol Biofuels 7:1-26. https://doi.org/10.1186/ 1754-6834-7-7

Johnson AO, Gonzalez-Villanueva M, Wong L, Steinbüchel A, Tee KL, Xu P, Wong TS (2017) Design and application of geneticallyencoded malonyl-CoA biosensors for metabolic engineering of microbial cell factories. Metab Eng 44:253-264. https://doi.org/10. 1016/j.ymben.2017.10.011 
Kallscheuer N, Marienhagen J (2018) Corynebacterium glutamicum as platform for the production of hydroxybenzoic acids. Microb Cell Factories 17:70. https://doi.org/10.1186/s12934-018-0923-x

Kallscheuer N, Vogt M, Stenzel A, Gätgens J, Bott M, Marienhagen J (2016) Construction of a Corynebacterium glutamicum platform strain for the production of stilbenes and $(2 S)$-flavanones. Metab Eng 38:47-55. https://doi.org/10.1016/j.ymben.2016.06.003

Kallscheuer N, Kage H, Milke L, Nett M, Marienhagen J (2019) Microbial synthesis of the type I polyketide 6-methylsalicylate with Corynebacterium glutamicum. Appl Microbiol Biotechnol 103: 9619-9631. https://doi.org/10.1007/s00253-019-10121-9

Khurana S, Venkataraman K, Hollingsworth A, Piche M, Tai TC (2013) Polyphenols: benefits to the cardiovascular system in health and in aging. Nutrients 5:3779-3827. https://doi.org/10.3390/nu5103779

Leonard E, Lim K-H, Saw P-N, Koffas MAG (2007) Engineering central metabolic pathways for high-level flavonoid production in Escherichia coli. Appl Environ Microbiol 73:3877-3886. https:// doi.org/10.1128/AEM.00200-07

Leonard E, Yan Y, Fowler ZL, Li Z, Lim CG, Lim KH, Koffas M (2008) Strain improvement of recombinant Escherichia coli for efficient production of plant flavonoids. Mol Pharm 5:257-265. https://doi. org $/ 10.1021 / \mathrm{mp} 7001472$

Li X, Guo D, Cheng Y, Zhu F, Deng Z, Liu T (2014) Overproduction of fatty acids in engineered Saccharomyces cerevisiae. Biotechnol Bioeng 111:1841-1852. https://doi.org/10.1002/bit.25239

Li S, Si T, Wang M, Zhao H (2015) Development of a synthetic malonylCoA sensor in Saccharomyces cerevisiae for intracellular metabolite monitoring and genetic screening. ACS Synth Biol 4:1308-1315. https://doi.org/10.1021/acssynbio.5b00069

Lian J, Si T, Nair NU, Zhao H (2014) Design and construction of acetylCoA overproducing Saccharomyces cerevisiae strains. Metab Eng 2:139-149. https://doi.org/10.1016/j.ymben.2014.05.010

Liang J, Guo L, Lin J, He Z, Cai F, Chen J (2016) A novel process for obtaining pinosylvin using combinatorial bioengineering in Escherichia coli. World J Microbiol Biotechnol 32:102. https://doi. org/10.1007/s11274-016-2062-z

Lim CG, Fowler ZL, Hueller T, Schaffer S, Koffas MAG (2011) High-yield resveratrol production in engineered Escherichia coli. Appl Environ Microbiol 77:3451-3460. https://doi.org/10.1128/AEM.02186-10

Marienhagen J, Bott M (2013) Metabolic engineering of microorganisms for the synthesis of plant natural products. J Biotechnol 163:166178. https://doi.org/10.1016/j.jbiotec.2012.06.001

Milke L, Aschenbrenner J, Marienhagen J, Kallscheuer N (2018) Production of plant-derived polyphenols in microorganisms: current state and perspectives. Appl Microbiol Biotechnol 102:1575-1585. https://doi.org/10.1007/s00253-018-8747-5

Milke L, Ferreira P, Kallscheuer N, Braga A, Vogt M, Kappelmann J, Oliveira J, Silva AR, Rocha I, Bott M, Noack S, Faria N, Marienhagen J (2019a) Modulation of the central carbon metabolism of Corynebacterium glutamicum improves malonyl-CoA availability and increases plant polyphenol synthesis. Biotechnol Bioeng 116:1380-1391. https://doi.org/10.1002/bit.26939

Milke L, Kallscheuer N, Kappelmann J, Marienhagen J (2019b) Tailoring Corynebacterium glutamicum towards increased malonyl-CoA availability for efficient synthesis of the plant pentaketide noreugenin. Microb Cell Factories 18:71. https://doi.org/10.1186/ s12934-019-1117-x

Milke L, Mutz M, Marienhagen J (2020) Synthesis of the character impact compound raspberry ketone and additional flavoring phenylbutanoids of biotechnological interest with Corynebacterium glutamicum. Microb Cell Factories 19:92. https://doi.org/10.1186/s12934-020-01351-y

Miyahisa I, Kaneko M, Funa N, Kawasaki H, Kojima H, Ohnishi Y, Horinouchi S (2005) Efficient production of (2S)-flavanones by Escherichia coli containing an artificial biosynthetic gene cluster.
Appl Microbiol Biotechnol 68:498-504. https://doi.org/10.1007/ s00253-005-1916-3

Nickel J, Irzik K, Van Ooyen J, Eggeling L (2010) The TetR-type transcriptional regulator FasR of Corynebacterium glutamicum controls genes of lipid synthesis during growth on acetate. Mol Microbiol 78: 253-265. https://doi.org/10.1111/j.1365-2958.2010.07337.x

Osbourn AE, Lanzotti V (2009) Plant-derived natural products - synthesis, function, and application. Springer New York, New York

Palmer CM, Alper HS (2018) Expanding the chemical palette of industrial microbes: metabolic engineering for type III PKS-derived polyketides. Biotechnol J 14:1700463. https://doi.org/10.1002/biot. 201700463

Pandey KB, Rizvi SI (2009) Plant polyphenols as dietary antioxidants in human health and disease. Oxidative Med Cell Longev 2:270-278. https://doi.org/10.4161/oxim.2.5.9498

Price AC, Choi K-H, Heath RJ, Li Z, White SW, Rock CO (2001) Inhibition of $\beta$-ketoacyl-acyl carrier protein synthases by thiolactomycin and cerulenin. J Biol Chem 276:6551-6559. https://doi.org/10.1074/jbc.M007101200

Rawlings BJ (2001) Type I polyketide biosynthesis in bacteria (part A erythromycin biosynthesis). Nat Prod Rep 18:190-227. https://doi. org $/ 10.1039 / \mathrm{b} 009329 \mathrm{~g}$

Robertsen HL, Musiol-Kroll EM (2019) Actinomycete-derived polyketides as a source of antibiotics and lead structures for the development of new antimicrobial drugs. Antibiotics 8:157. https://doi.org/10.3390/antibiotics8040157

Santos CNS, Koffas M, Stephanopoulos G (2011) Optimization of a heterologous pathway for the production of flavonoids from glucose. Metab Eng 13:392-400. https://doi.org/10.1016/j.ymben. 2011.02.002

Schujman GE, Guerin M, Buschiazzo A, Schaeffer F, Llarrull LI, Reh G, Vila AJ, Alzari PM, de Mendoza D (2006) Structural basis of lipid biosynthesis regulation in Gram-positive bacteria. EMBO J 25: 4074-4083. https://doi.org/10.1038/sj.emboj.7601284

Sheng J, Feng X (2015) Metabolic engineering of yeast to produce fatty acid-derived biofuels: bottlenecks and solutions. Front Microbiol 6: 554. https://doi.org/10.3389/fmicb.2015.00554

Shi S, Chen Y, Siewers V, Nielsen J (2014) Improving production of malonyl coenzyme A-derived metabolites by abolishing Snf1dependent regulation of Acc1. MBio 5:e01130-e01114. https://doi. org/10.1128/mBio.01130-14

Shimokawa Y, Morita H, Abe I (2012) Benzalacetone synthase. Front Plant Sci 3:57. https://doi.org/10.3389/fpls.2012.00057

Stratistics Market Research Consulting Pvt Ltd (2018) Nutraceuticals global market outlook (2017-2026). In: Nutraceuticals - Glob. Mark. Outlook. https://www.strategymrc.com/report/nutraceuticalsmarket-2018. Accessed 7 Jan 2020

Takeno S, Takasaki M, Urabayashi A, Mimura A, Muramatsu T, Mitsuhashi S, Ikeda M (2013) Development of fatty acidproducing Corynebacterium glutamicum strains. Appl Environ Microbiol 79:6776-6783. https://doi.org/10.1128/AEM.02003-13

Tong L (2005) Acetyl-coenzyme A carboxylase: crucial metabolic enzyme and attractive target for drug discovery. Cell Mol Life Sci 62:1784-1803. https://doi.org/10.1007/s00018-005-5121-4

van Summeren-Wesenhagen PV, Marienhagen J (2015) Metabolic engineering of Escherichia coli for the synthesis of the plant polyphenol pinosylvin. Appl Environ Microbiol 81:840-849. https://doi.org/10. 1128/AEM.02966-14

Wang Y, Chen H, Yu O (2014) A plant malonyl-CoA synthetase enhances lipid content and polyketide yield in yeast cells. Appl Microbiol Biotechnol 98:5435-5447. https://doi.org/10.1007/s00253-014-5612-z

Wattanachaisaereekul S, Lantz AE, Nielsen ML, Nielsen J (2008) Production of the polyketide 6-MSA in yeast engineered for increased malonyl-CoA supply. Metab Eng 10:246-254. https://doi. org/10.1016/j.ymben.2008.04.005 
Weissman KJ, Leadlay PF (2005) Combinatorial biosynthesis of reduced polyketides. Nat Rev Microbiol 3:925-936. https://doi.org/10.1038/ nrmicro1287

Wink M (ed) (2010) Functions and biotechnology of plant secondary metabolites, second. Wiley-Blackwell, West Sussex

Wu J, Du G, Zhou J, Chen J (2013) Metabolic engineering of Escherichia coli for (2S)-pinocembrin production from glucose by a modular metabolic strategy. Metab Eng 16:48-55. https://doi.org/10.1016/j. ymben.2012.11.009

Wu J, Yu O, Du G, Zhou J, Chen J (2014) Fine-tuning of the fatty acid pathway by synthetic antisense RNA for enhanced (2S)-naringenin production from L-tyrosine in Escherichia coli. Appl Environ Microbiol 80:7283-7292. https://doi.org/10.1128/AEM.02411-14

Wu J, Du G, Chen J, Zhou J (2015) Enhancing flavonoid production by systematically tuning the central metabolic pathways based on a CRISPR interference system in Escherichia coli. Sci Rep 5:13477. https://doi.org/10.1038/srep13477

Wu G, Yan Q, Jones JA, Tang YJ, Fong SS, Koffas MAG (2016) Metabolic burden: cornerstones in synthetic biology and metabolic engineering applications. Trends Biotechnol 34:652-664. https:// doi.org/10.1016/j.tibtech.2016.02.010

Wu J, Zhang X, Xia X, Dong M (2017) A systematic optimization of medium chain fatty acid biosynthesis via the reverse beta-oxidation cycle in Escherichia coli. Metab Eng 41:115-124. https://doi.org/ 10.1016/j.ymben.2017.03.012

Xu P, Ranganathan S, Fowler ZL, Maranas CD, Koffas MAG (2011) Genome-scale metabolic network modeling results in minimal interventions that cooperatively force carbon flux towards malonyl-CoA. Metab Eng 13:578-587. https://doi.org/10.1016/j.ymben.2011.06.008
Xu P, Li L, Zhang F, Stephanopoulos G, Koffas M (2014a) Improving fatty acids production by engineering dynamic pathway regulation and metabolic control. Proc Natl Acad Sci U S A 111:11299-11304. https://doi.org/10.1073/pnas.1406401111

Xu P, Wang W, Li L, Bhan N, Zhang F, Koffas MAG (2014b) Design and kinetic analysis of a hybrid promoter-regulator system for malonylCoA sensing in Escherichia coli. ACS Chem Biol 9:451-458. https://doi.org/10.1021/cb400623m

Yang Y, Lin Y, Li L, Linhardt RJ, Yan Y (2015) Regulating malonyl-CoA metabolism via synthetic antisense RNAs for enhanced biosynthesis of natural products. Metab Eng 29:217-226. https://doi.org/10. 1016/j.ymben.2015.03.018

Yang D, Kim WJ, Yoo SM, Choi JH, Ha SH, Lee MH, Lee SY (2018) Repurposing type III polyketide synthase as a malonyl-CoA biosensor for metabolic engineering in bacteria. Proc Natl Acad Sci U S A 115:9835-9844. https://doi.org/10.1073/pnas.1808567115

Zha W, Rubin-Pitel SB, Shao Z, Zhao H (2009) Improving cellular malonylCoA level in Escherichia coli via metabolic engineering. Metab Eng 11: 192-198. https://doi.org/10.1016/j.ymben.2009.01.005

Zhang S, Yang W, Chen H, Liu B, Lin B, Tao Y (2019) Metabolic engineering for efficient supply of acetyl-CoA from different carbon sources in Escherichia coli. Microb Cell Factories 18:130. https:// doi.org/10.1186/s12934-019-1177-y

Publisher's note Springer Nature remains neutral with regard to jurisdictional claims in published maps and institutional affiliations. 